\title{
Decreased public pursuit of cancer-related information during the COVID-19 pandemic in the United States
}

\author{
Siegfried Adelhoefer ${ }^{1,2} \cdot$ Philipp Berning ${ }^{3} \cdot$ Stephen B. Solomon ${ }^{4} \cdot$ Majid Maybody $^{4} \cdot$ Seamus P. Whelton ${ }^{1}$. \\ Michael J. Blaha ${ }^{1} \cdot$ Omar Dzaye ${ }^{1,2,5}$
}

Received: 20 October 2020 / Accepted: 25 February 2021 / Published online: 8 March 2021

(c) The Author(s), under exclusive licence to Springer Nature Switzerland AG 2021

\begin{abstract}
Background In response to the prioritization of healthcare resources towards the COVID-19 pandemic, routine cancer screening and diagnostic have been disrupted, potentially explaining the apparent COVID-era decline in cancer cases and mortality. In this study, we identified temporal trends in public interest in cancer-related health information using the nowcasting tool Google Trends.

Methods We used Google Trends to query search terms related to cancer types for short-term (September 2019-September 2020) and long-term (September 2016-September 2020) trends in the US. We compared average relative search volumes (RSV) for specified time ranges to detect recent and seasonal variation.

Results General search interest declined for all cancer types beginning in March 2020, with changes in search interest for "Breast cancer," "Colorectal cancer," and "Melanoma" of $-30.6 \%,-28.2 \%$, and $-26.7 \%$, respectively, and compared with the mean RSV of the two previous months. In the same time range, search interest for "Telemedicine" has increased by $+907.1 \%$ and has reached a 4-year peak with a sustained increased level of search interest. Absolute cancer mortality has declined and is presently at a 4-year low; however, search interest in cancer has been recuperating since July 2020.

Conclusion We observed a marked decline in searches for cancer-related health information that mirrors the reduction in new cancer diagnoses and cancer mortality during the COVID-19 pandemic. Health professions need to be prepared for the coming demand for cancer-related healthcare, foreshadowed by recovering interest in cancer-related information on Google Trends.
\end{abstract}

Keywords Infodemiology $\cdot$ Google trends $\cdot$ Health promotion $\cdot$ Patient education $\cdot$ Cancer awareness

Omar Dzaye

odzaye@jhmi.edu

1 Johns Hopkins Ciccarone Center for Prevention of Cardiovascular Disease, The Johns Hopkins Hospital, Johns Hopkins University School of Medicine, Blalock 524D1, 600 N Wolfe St, Baltimore, MD 21287, USA

2 Department of Radiology and Neuroradiology, Charité, Berlin, Germany

3 Department of Hematology and Oncology, University Hospital Muenster, Muenster, Germany

4 Department of Radiology, Interventional Radiology Service, Memorial Sloan Kettering Cancer Center, New York, NY, USA

5 Russell H. Morgan Department of Radiology and Radiological Science, Johns Hopkins University School of Medicine, Baltimore, MD, USA

$\begin{array}{ll}\text { Abbreviations } \\ \text { AML } & \text { Acute myeloid leukemia } \\ \text { CRC } & \text { Colorectal cancer } \\ \text { HCC } & \text { Hepatocellular carcinoma } \\ \text { NCHS } & \text { National Center for Health Statistics } \\ \text { NSCLC } & \text { Non-small cell lung cancer } \\ \text { PSA } & \text { Prostate-specific antigen } \\ \text { US } & \text { United States } \\ \text { RSV } & \text { Relative search volume } \\ \text { SCLC } & \text { Small-cell lung cancer } \\ \text { SVI } & \text { Search volume index }\end{array}$

\section{Introduction}

The ongoing coronavirus disease 2019 (COVID-19) pandemic poses several challenges for cancer care [1]. Patients with cancer are more susceptible to severe complications 
of COVID-19 due to their immunosuppressed status, caused both directly by cancer and from cancer treatment [2-4]. Although many cancers require timely diagnosis and treatment, healthcare providers have had to delay or cancel important activities including screening programs, surgery, chemotherapy, and radiation therapy.

While numbers in surgeries and admissions to emergency departments decreased, a substantial increase in telemedicine has been reported [5, 6]. As a tool to minimize exposure to SARS-CoV-2 for both patients and healthcare providers, telehealth has been shown to be a feasible alternative in the management of chronic diseases such as diabetes and has recently been adopted in the guidelines of the American Society of Clinical Oncology (ASCO) for cancer patients not urgently requiring a physical exam, treatment, or diagnostics [6-9].

The beneficial role of seeking cancer-related information especially for the adherence to preventive measures including cancer screening and cancer risk factor optimization has been shown in several observational studies [10, 11]. Additionally, a clear increase in health-related and life quality in cancer survivors that actively seek cancerrelated information has been previously reported [12, 13]. It is, however, less well known to what extent the ongoing COVID-19 pandemic may have influenced these potentially life-saving search behaviors. In line with the known phenomena of redirected healthcare resources in response to the ongoing pandemic, online search interests for COVID-19-related information peaked with increasing COVID-19 case numbers [14]. This might in turn suggest a diversion of typical cancer-related online search behavior. The analyses of the distribution of online public health data have sparked the formation of the rather new field termed infodemiology [15]. Infodemiology has contributed to health evaluation by assessing real-time (online) information in a range of diseases and conditions including infectious diseases and cardiovascular health [16-19]. Google Trends represents a publicly available database allowing to monitor searches on the commonly used Google search engine in near real time [19]. It has been shown to potentially complement epidemiologic trends in cancer incidences and cancer screening for the US [20,21].

In this study, we aim to explore short-term and long-term trends in public online search interest for the most common cancer entities according to recent cancer statistics. We hypothesized a temporal decrease in search interest following the official declaration of the global COVID19 pandemic as well as the imposed lockdown measures across numerous US states throughout March 2020. We further hypothesized a gradual recuperation of cancer-related online search volumes with lockdown measures being lifted in May-June 2020. In response to the COIVD-19 crisis management, we expected a relative and transient increase in search behavior related to telemedicine.

\section{Methods}

Google Trends (https://trends.google.com/) is a publicly available query tool that aggregates data on Google search engine trends, both—geographically and over time. Based on most recent epidemiological estimates on prevailing cancer types [22], we queried the most common cancer types and topographic anatomic regions, including cervical cancer, hepatocellular carcinoma, melanoma, bladder cancer, thyroid cancer, multiple myeloma, meningioma, and acute myeloid leukemia. Additionally, we grouped several search terms into a subset of cancer types associated with modifiable risk factors (including unhealthy diet, obesity, physical inactivity, and tobacco use), namely small-cell lung cancer (SCLC), non-small cell lung cancer (NSCLC), and colorectal cancer. We also grouped search terms into a subset of cancer types considered hormone sensitive including breast cancer, ovarian cancer, endometrial cancer, uterine cancer, and prostate cancer. Topographic regions included cancers of the digestive system (averaging search volume for "Esophageal cancer," "Gallbladder cancer," "Pancreatic cancer," "Hepatocellular carcinoma," and "Colorectal cancer"), respiratory system (averaging search volume for "Laryngeal cancer," "Small-cell lung carcinoma," and "Non-small cell carcinoma"), breast and genital system (averaging search volume for "Breast cancer," "Ovarian cancer," "Endometrial cancer," "Uterine cancer," "Prostate cancer," and "Cervical cancer"), urinary system (averaging search volume for "Renal cell carcinoma" and "Bladder cancer"), and head and neck region (averaging search volume for "Oropharyngeal cancer," "Oral cancer," "Nasopharyngeal cancer," "Astrocytoma," "Glioblastoma," "Thyroid cancer," and "Meningioma"). We queried all terms using search categories assigned by Google; these included "Topic," "Disease," or "Medical condition" (Supplemental Table 1). We used these search categories to encompass a broader range of search results that were queried with a similar intention; we, therefore, chose search terms Google identified as most commonly searched over the medically more common term-the search term "Small-cell carcinoma" includes searches for "small-cell lung carcinoma." Details on the decision making behind this assignment process are described by Google Trends in more detail [23]. We also used Google's "near me"-tool, a suffix used for searches in Google and affiliated websites to show customized and customer behavior-oriented search queries. Search terms queried with the addition of "near me" were considered indicative for a potential interest in medical services in the 
near future and vicinity. We used this tool for the search term "Doctor near me." Additionally, we queried search terms related to telehealth including "Telehealth," "Telemedicine," and "Virtual doctor visit."

We defined two time ranges: short-term trends were defined by the time range of September 1, 2019 to September 1, 2020, long-term trends as the time range September 1, 2016 to September 1, 2020. Data included in this analysis represent search volume in the US to reduce potential variability between strategic approaches related to COVID19 in different countries. All data for further quantitative analysis were extracted on September 21, 2020. Google reports search trends as relative search volume (RSV), i.e., all exported values are relative to the peak search volume index (SVI) of 100 in for the indicated time range and location. Thus, singular events with a sudden peak in search interest may make the SVI at all other time points appear low-this has previously been reported for events that draw significant public attention, as for example the National Breast Cancer Awareness Month [24]. Search terms were excluded from this study if we detected such sudden spikes lasting less than three consecutive weeks and recuperating to prior levels within that time range; if feasible, we excluded only a limited time range to mirror search interest for the rest of the time range specified above. Of note, the included data from Google Trends solely represent search terms queried through Google and affiliated websites but does not include other search engines. This study utilized publicly available data without personal identifiers and was, thus, exempt from institutional review board approval.

\section{Results}

\section{Decrease in online search interest at the beginning of 2020}

For all cancer types included in our analysis, online search interest via Google and affiliated search websites results declined sharply since March 2020. Search interest changed for the terms "Cervical cancer" $(-30.0 \%)$, "Hepatocellular carcinoma" (-21.8\%), "Melanoma" (-26.7\%), "Thyroid cancer" $(-33.9 \%)$, and "Meningioma" $(-38.0 \%)$, when comparing the mean RSV of March-April 2020 with the mean RSV of the previous two months. We noted a rebound phenomenon that varied across cancer types. For example, search interest increased moderately for "Cervical cancer" $(+8.9 \%)$ and "Hepatocellular carcinoma" $(+2.8 \%)$ and stronger for "Melanoma" $(+26.6 \%)$ and "Meningioma" (+15.2\%), when comparing the mean RSV of May-June 2020 with the mean RSV of the two preceding months (Fig. 1a, b; Supplemental Table 2).

\section{Search interest among subgroups of cancer depending on risk factor profiles}

Based on the underlying risk factor profiles, cancers that are largely attributable to modifiable risk factors (colorectal cancer and lung cancer) and sex-specific cancers (breast, ovarian, endometrial, uterine, and prostate cancer) are depicted separately (Figs. 2, 3) [25]. These cancer types exhibit more options for primary and secondary prevention and may, thus, depend even more on in-person clinical visits to either avoid (further) progression or enhance overall outcome. Lifestyle and dietary changes are also crucial in some of these types. For cancer entities related to modifiable risk factors, we observed a similar trend as in Fig. 1. Search interest decreased for "Small-cell lung carcinoma" $(-30.7 \%)$, "Non-small cell lung cancer" $(-22.1 \%)$ and "Colorectal cancer" $(-28.2 \%)$, when comparing the RSV means of January-February 2020 to March-April 2020. Search interest has since partly recuperated with changes of $-0.7 \%,+7.3 \%$, and $+10.0 \%$, respectively, comparing May-June 2020 with the preceding 2 months (Fig. 2a, Supplemental Table 3).

For sex-specific cancers, search interest decreased since March 2020 reaching its minimum at the end of March 2020. Similar to modifiable risk-related cancers, search interest has been recuperating since April 2020 (Fig. 3a, Supplemental Table 4).

Interestingly, for "Breast cancer," a strong peak in October 2019 was observed that was consistently to be detected in the defined long-term timeframe from September 1, 2016 to September 1, 2020, potentially reflecting a seasonal pattern (Fig. 3b). This finding largely corresponds with the National Breast Cancer Awareness Month every October. Also, but less strikingly, a similar pattern could be detected for "Ovarian cancer" in September, and "Endometrial cancer" in November of each year (Fig. 3b). Additionally, we identified seasonal peaks for "Non-small cell lung cancer" in February and for "Colorectal cancer" in February/March annually (Fig. 2b).

In order to even out the observed seasonal peaks in search volumes for individual cancer entities, the respective entities were subsumed according to the affected organ system or region. As shown in Supplemental Fig. 1A, search volumes clearly dropped in March 2020 and showed a rising trend in May-June with a subsequent recuperation. Moreover, we confirm that the decline in search interest as seen in March 2020 was unprecedented in the 4-year time scale (Supplemental Fig. 1B).

\section{Face-to-face and remote patient-doctor interaction}

Online search volumes for "Telehealth," "Telemedicine," and "Virtual doctor visit" changed 

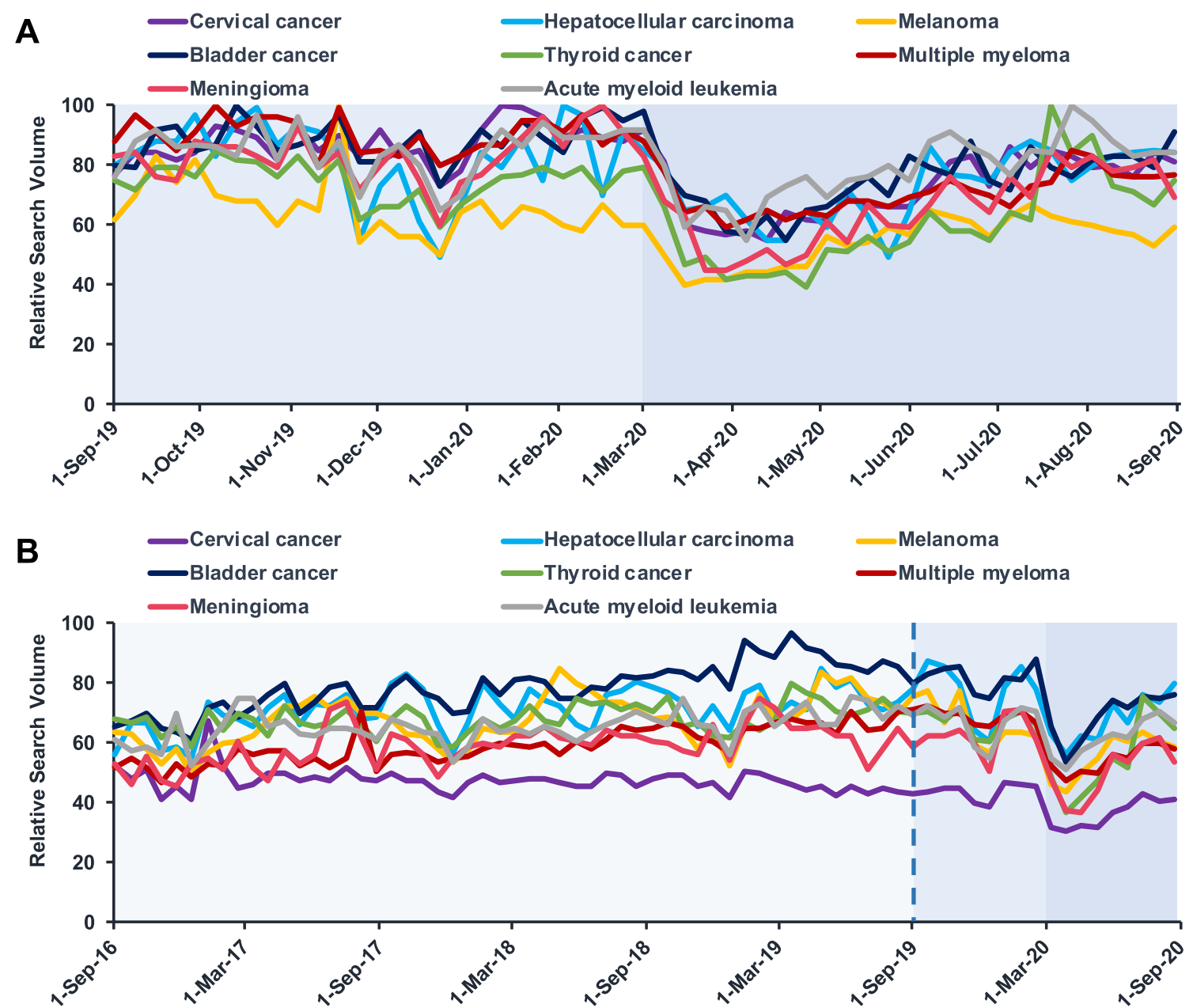

Fig. 1 US online search interest in a set of common cancer types. Google Trends relative search volume during the time periods a September 1, 2019 to September 1, 2020 presented as weekly data and b September 1, 2016 to September 1, 2020 presented as tri-weekly data for the search terms "Cervical cancer" (purple), "Hepatocellular carcinoma" (light blue), "Melanoma" (yellow), "Bladder cancer" (dark blue), "Thyroid cancer" (green), "Multiple myeloma" (dark red), "Meningioma" (pink), and "Acute myeloid leukemia" (gray). Shaded area: World Health Organization (WHO) on March 11, 2020 has declared the COVID-19 outbreak a global pandemic. Dashed line in lower panel implicates start of upper panel to visualize transient trends amidst COVID-19 pandemic versus long-term seasonal trends. (Search query: 09/20/2020). (Color figure online) by $+1047.1 \%,+907.1 \%$, and $+421.9 \%$, respectively, when comparing the mean RSV of September 2019-February 2020 with the mean RSV of March 2020. In the same time range, interest for the search term "Doctor near me" declined by $-23.3 \%$ (Fig. 4a, Supplemental Table 5). However, in mid-April, these trends reversed; search volumes for "Doctor near me" increased in the following three months after March $2020(+13.3 \%)$ and were at a four-year high by July 1, 2020 (Fig. 4b). Conversely, search interest in telehealth-related terms has plummeted since April, but search volume remains still substantially higher than before March 1, 2020.

\section{Discussion}

Concordant with emerging warnings on delayed diagnostic and therapeutic procedures of patients with cancer, we report a decline in online search volumes for cancerrelated information. The online search for cancer-related information is often the first approach for individuals to contextualize potential symptoms or concerns that do not require immediate emergency care. There are several potential explanations for this decrease. First, patients with non-specific symptoms may be reluctant to seek medical 
A

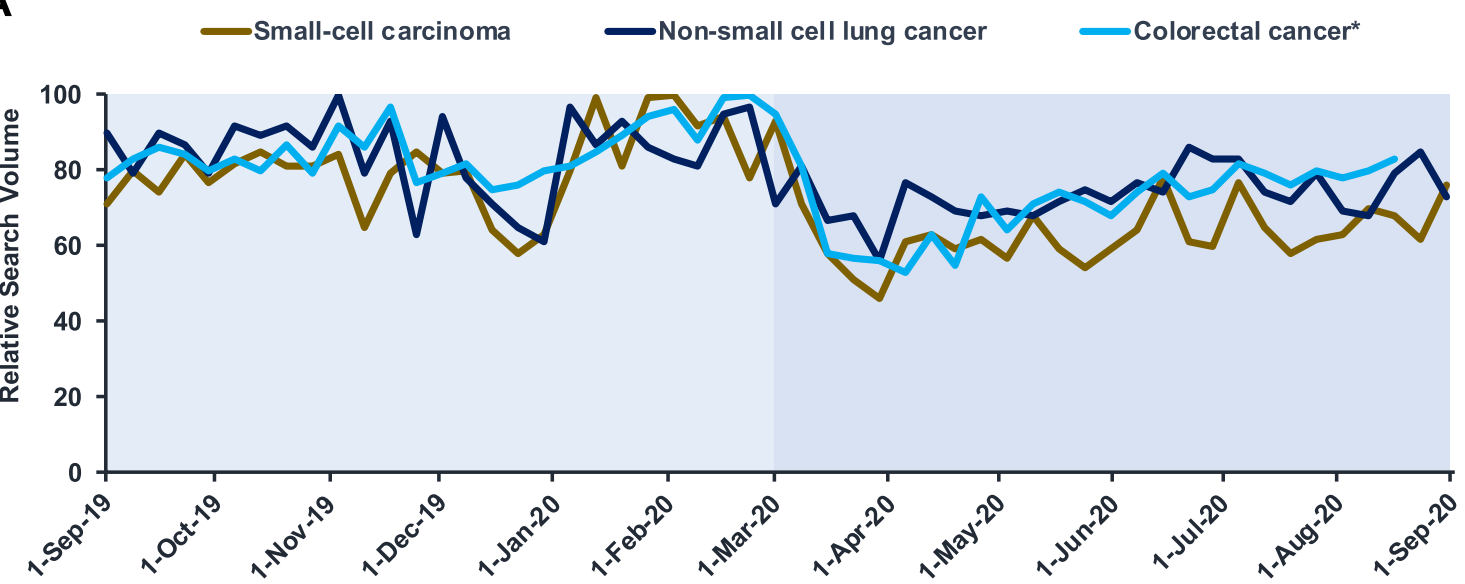

B

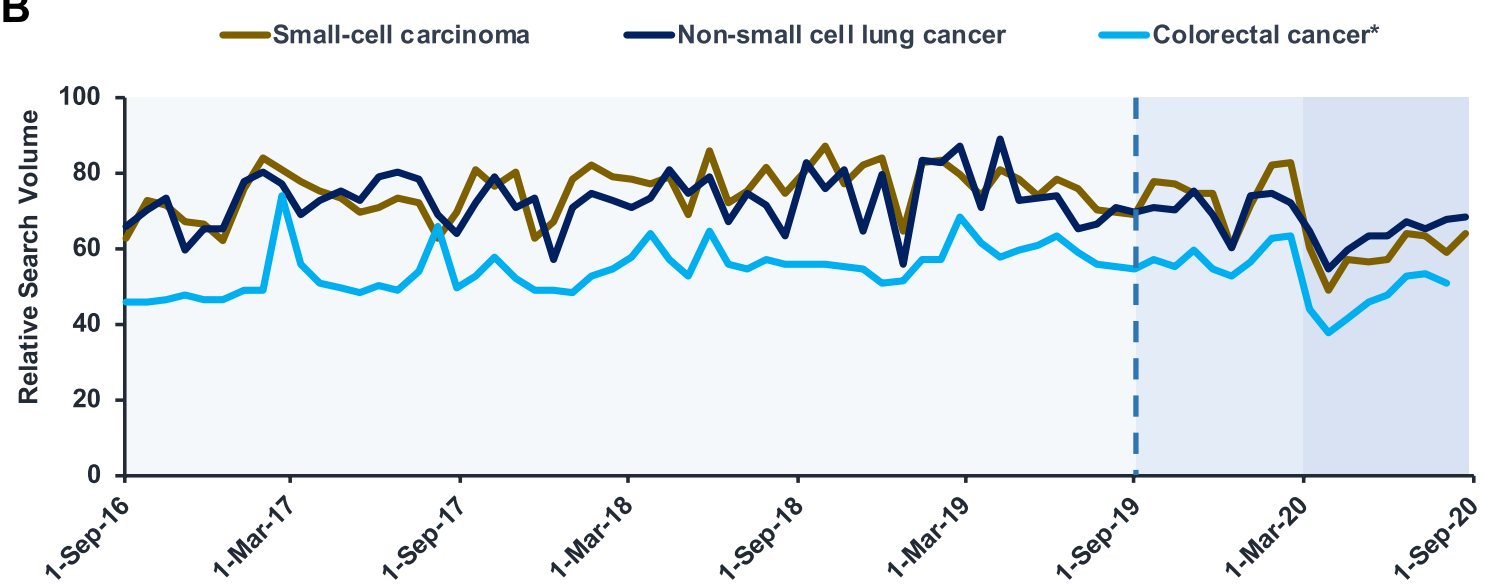

Fig. 2 US online search interest in terms related to cancer types attributable to modifiable risk factors. Google Trends relative search volume during the time periods a September 1, 2019 to September 1, 2020 presented as weekly data and b September 1, 2016 to September 1, 2020 presented as tri-weekly data for the search terms "Small-cell carcinoma" (brown), "Non-small cell lung cancer" (dark blue), and "Colorectal cancer" (light blue); the Google search term "Small-cell carcinoma" includes searches for "small-cell lung cancer." *For the

help for fear of acquiring SARS-CoV-2 in a healthcare setting. Second, hospitals and healthcare providers have postponed diagnostic procedures potentially leading to a decreased pursuit of non-COVID-19-related information online. Third, established screening programs for breast, colon and cervical cancer have been paused in order to alleviate the impact of the pandemic on the healthcare system.

Contrary to the recently stagnating mortality rates in many cancer types [22], the widespread postponement of diagnosis and treatment may ultimately result in an increase in cancer mortality rates. Public de-prioritization of cancer care as reflected by decreased public online search interest could lead to future fatal consequences of cancer and may impose a future economic burden on the healthcare system search term "Colorectal cancer," we excluded the dates from August 23, 2020 onwards due to a spike in search interest possibly following the death of Chadwick Boseman from CRC; see Supplemental Fig. 2. Shaded area: World Health Organization (WHO) on March 11, 2020 has declared the COVID-19 outbreak a global pandemic. Dashed line in lower panel implicates start of upper panel to visualize transient trends amidst COVID-19 pandemic versus long-term seasonal trends. (Search query: 09/20/2020). (Color figure online)

[26]. However, as we observed recurrent annual increases for months considered as specific cancer awareness months, public interest in cancer-related terms is likely to be mirrored by online search volumes. For instance, besides the National Breast Cancer Awareness Month March, recurrent short-term increases were also noted for ovarian and colorectal cancer that corresponded with the respective awareness months. These findings further illustrate that online search behavior might represent an additional tool for public health communication strategies in cancer care.

\section{Cancer patients and COVID-19}

Several reports show an increased mortality for cancer patients due to COVID-19, irrespective of whether they are 
A Breast cancer $\longrightarrow$ Ovarian cancer - Endometrial cancer $\longrightarrow$ Uterine cancer $\longrightarrow$ Prostate cancer

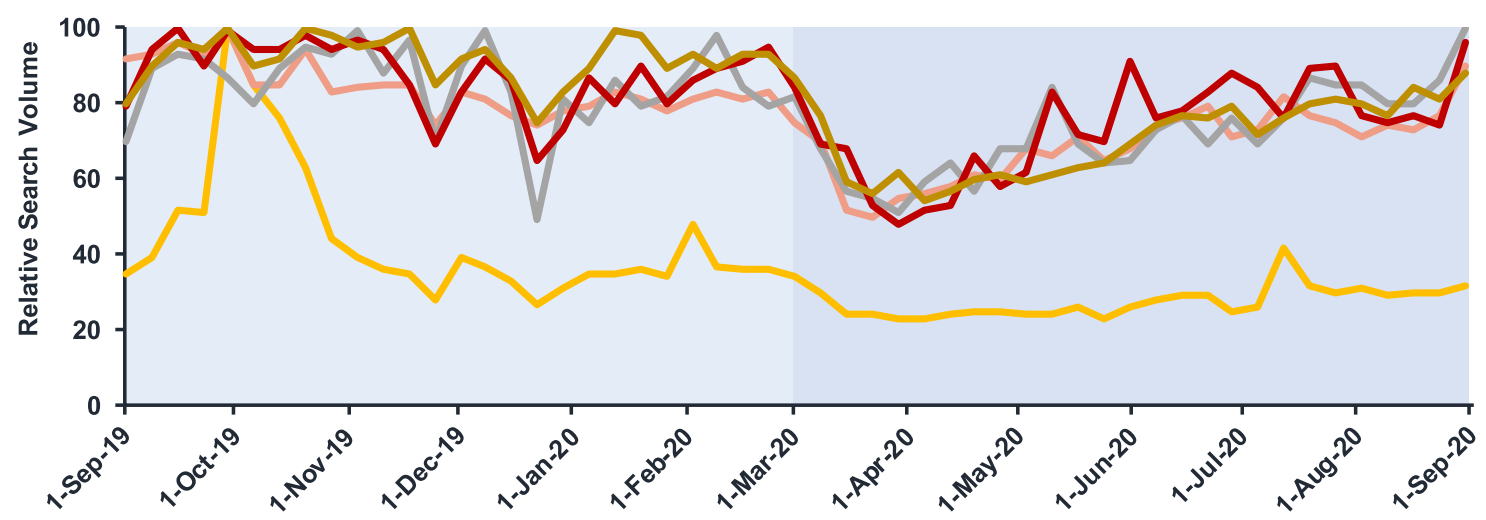

B
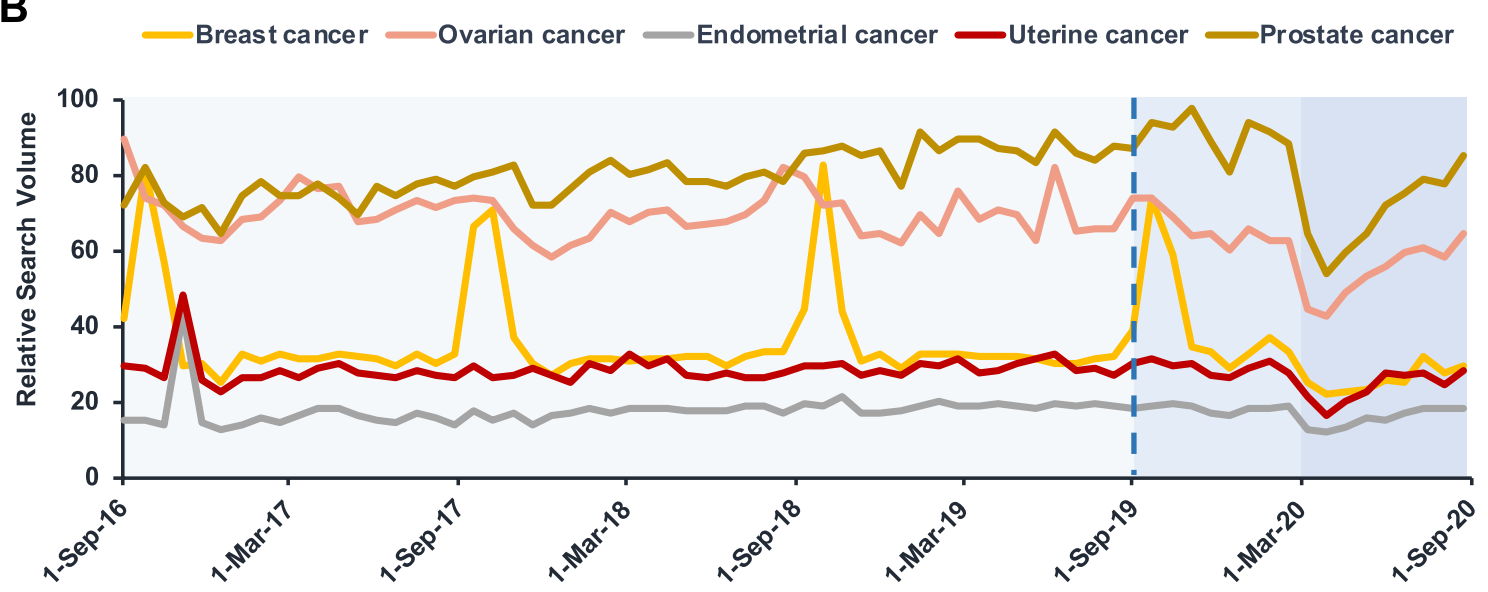

Fig. 3 US online search interest in a set of sex-specific cancer types. Google Trends relative search volume during the time periods a September 1, 2019 to September 1, 2020 presented as weekly data and b September 1, 2016 to September 1, 2020 presented as tri-weekly data for the search terms "Breast cancer" (yellow), "Ovarian cancer" (rose), "Endometrial cancer" (gray), "Uterine cancer" (dark red), and
"Prostate cancer" (light brown). Shaded area: World Health Organization (WHO) on March 11, 2020 has declared the COVID-19 outbreak a global pandemic. Dashed line in lower panel implicates start of upper panel to visualize transient trends amidst COVID-19 pandemic versus long-term seasonal trends. (Search query: 09/20/2020). (Color figure online) receiving anticancer therapies or have active cancer diseases [27-29]. However, Mei et al. based on their experience from the Wuhan Union Hospital, identified infections acquired in an in-patient setting as one major threat to cancer patients in the beginning recent pandemic [3, 30]. According to the National Center for Health Statistics (NCHS) datasets, US cancer mortality rates are continuously declining due to treatment breakthroughs and improved risk reduction strategies [22]. Despite the seasonal variability that must be taken into account, cancer mortality fell to a four-year low in May 2020 according to our cursory analysis of the available NCHS cancer statistics. Thus, delivering cancer care to patients during this pandemic remains one of the most urgent challenges and requires a reasonable balancing of competing risks of death and morbidity from cancer versus death from COVID-19 [31]. Conversely, changes in health-seeking behavior that is likely to have beneficial effects on cancer outcomes [10-13] as well as a transiently limited availability of essential diagnostics and care modalities in the course of the pandemic have been observed. Newly diagnosed cancer cases for six common cancer types have declined by $46.4 \%$ soon after the pandemic unfolded [32]. Recent registrybased analyses from the UK implicate that the experienced delays in cancer diagnostics and treatment, especially cancer surgery, most probably will lead to a collateral increase in avoidable cancer deaths of various cancer entities [33, 34].

\section{Cancer types with modifiable risk factors}

The decline in search interest for cancer types attributable to modifiable risk factors, which most likely could profit from preventive efforts, may imply decreased public awareness 
A

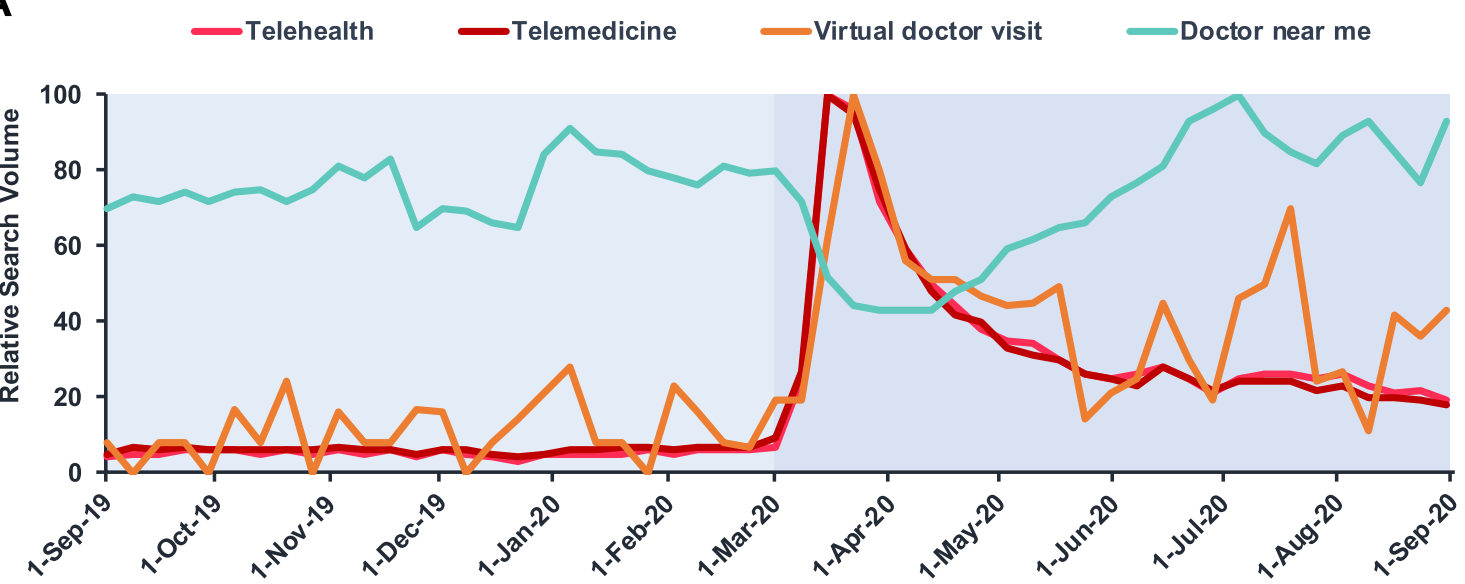

B
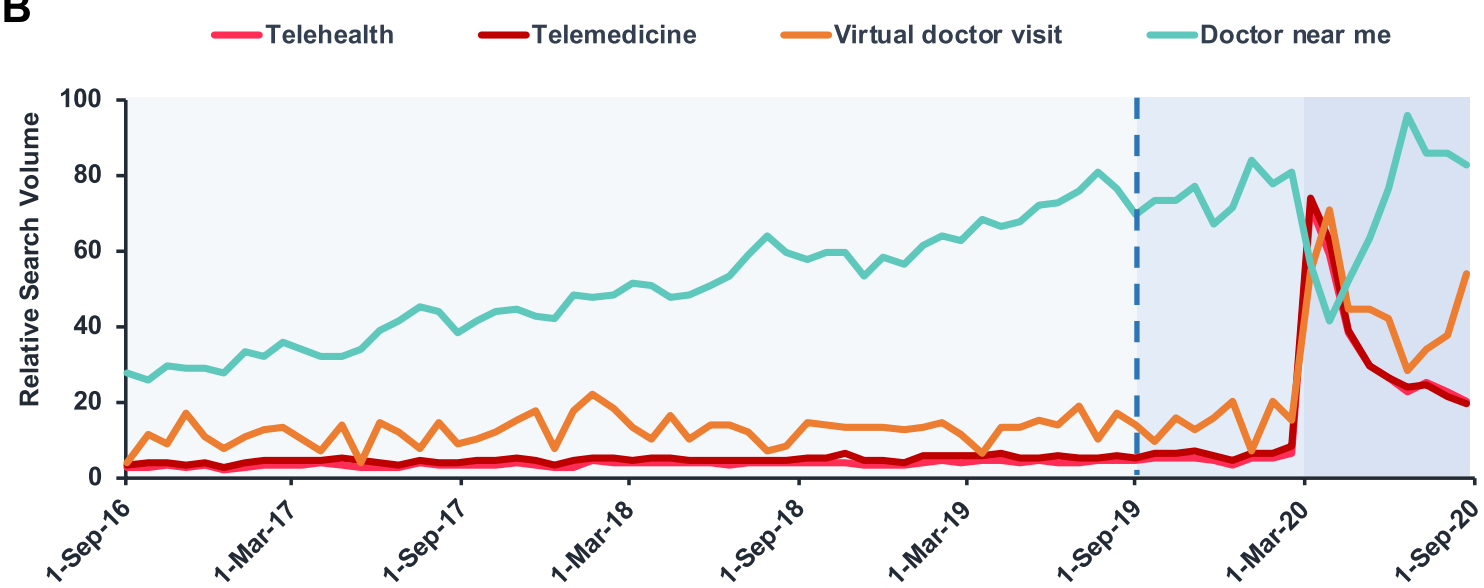

Fig. 4 US online search interest in telehealth and related terms. Google Trends relative search volume (RSV) during the time periods a September 1, 2019 to September 1, 2020 presented as weekly data and b September 1, 2016 to September 1, 2020 presented as triweekly data for the search terms "Telehealth" (pink), "Telemedicine" (dark red), "Virtual doctor visit" (orange), and "Doctor near me" (tur- quoise). Shaded area: World Health Organization (WHO) on March 11, 2020 has declared the COVID-19 outbreak a global pandemic. Dashed line in lower panel implicates start of upper panel to visualize transient trends amidst COVID-19 pandemic versus long-term seasonal trends. (Search query: 09/20/2020). (Color figure online)

timely diagnostics and possibly surgery to achieve cure or symptom control. However, these patients are at a particular risk for a serious course of COVID-19 due to their cancer as well as the respective treatment [37].

\section{The shift towards telehealth}

In light of the rapid growth in telehealth, our findings on telehealth-related terms support a clear trend towards a public interest in virtual healthcare [3]. Telemedicine can partly substitute the patient-doctor interaction for the short term $[7,8]$. However, it cannot replace crucial steps in the diagnostic and treatment process of cancers. For the duration of the pandemic, telehealth tools are recommended by society guidelines as summarized elsewhere [7, 9]. There are, however, limitations of this technology: systemic barriers and chest pain. Eventually, lung cancer treatment requires 
including funding and legal concerns, acceptance by patients and doctors, continuous training for the effective usage, and broad accessibility of technical infrastructure. As an end of the preventive measures against COVID-19 remains unlikely in the short-term future, telemedicine can contribute to providing needed care for cancer patients, potentially even with a high degree of consistency in the patient-doctor relationship [5, 38].

\section{Preparing for an increase in cancer patients}

We also showed a recent recuperation of online search interest for various cancer entities, which might indicate for an increase in demand for cancer care.

Nonetheless, the observed decrease in cancer mortality revealed by NCHS statistics might mirror the pandemicrelated changes in healthcare behavior and delays in diagnostics and treatments. Thus, policy interventions are required to address the potential increase in collateral effects of COVID-19 for cancer diagnostics and treatments. As the vast online search interest in cancer-related search terms has not yet reached its pre-pandemic degree, public health messaging sensitizing, and conveying the risks of delayed cancer diagnostics, screenings and therapy versus COVID-19 could be considered. Furthermore, clear recommendations guiding clinicians in the decision making for prioritization of high-risk patients as well in the management of required procedures in cancer care are required.

\section{Limitations}

The limitations of our study are a potential overrepresentation of internet users, most likely younger individuals, actively searching for health-related terms. Second, we cannot confirm that all search activity for the respective terms exclusively reflects health-seeking behavior. The data presented complement but do not substitute for traditional epidemiological studies or analyses of actual clinical activity. Third, as Google Trends does not provide information on the individual who is seeking information, we could not identify specific subgroups with a potentially higher share in search volumes. As such, potential subgroups of interest might be the general public, cancer patients under treatment, cancer survivors, and healthcare professionals. Lastly, data from Google Trends may not represent part of the general public without access to the internet.

\section{Conclusions}

Online search interests of cancer-related screening/diagnostic information have experienced a sharp decline since the beginning of the COVID-19 pandemic in the United States.
Early evidence suggests a decline in newly diagnosed cancer cases and a decline in cancer mortality. However, we observed an increase in online search interest in recent weeks potentially translating into an increased demand for cancer care in upcoming months. Delayed diagnostic and therapeutic procedures may require mechanisms of prioritization to alleviate healthcare resources and yet, reduce an inevitable rise in cancer mortality.

Supplementary Information The online version contains supplementary material available at https://doi.org/10.1007/s10552-021-01409-1.

Author contributions OD, SA, and MJB participated in the conception and design of the study, and drafted the manuscript. OD and SA conducted the analyses and prepared the tables and figures. PB, SBS, $\mathrm{MM}$, and SPW participated in the interpretation of the data, drafting of the manuscript, and revised subsequent drafts critically for important intellectual content. All authors approved the final version.

Funding Not applicable.

\section{Declarations}

Conflict of interest The authors declare that they have no conflicts of interest relevant to the content of this manuscript.

Ethical approval This study utilized publicly available data without personal identifiers and was, thus, exempt from institutional review board approval.

\section{References}

1. Pennell NA, Dillmon M, Levit LA et al (2020) American Society of Clinical Oncology Road to Recovery Report: learning From the COVID-19 experience to improve clinical research and cancer care. J Clin Oncol 39:155-169

2. Dai M, Liu D, Liu M et al (2020) Patients with cancer appear more vulnerable to SARS-COV-2: a multicenter study during the COVID-19 outbreak. Cancer Discov 10:783-791

3. Mei H, Dong X, Wang Y, Tang L, Hu Y (2020) Managing patients with cancer during the COVID-19 pandemic: frontline experience from Wuhan. Lancet Oncol 21:634-636

4. Miyashita H, Mikami T, Chopra N et al (2020) Do patients with cancer have a poorer prognosis of COVID-19? An experience in New York City. Ann Oncol 31:1088-1089

5. Bashshur RL, Doarn CR, Frenk JM, Kvedar JC, Shannon GW, Woolliscroft JO (2020) Beyond the COVID pandemic, telemedicine, and health care. Telemed J e-Health 26:1310-1313

6. Dosaj A, Thiyagarajan D, Ter Haar C et al (2020) Rapid implementation of telehealth services during the COVID-19 pandemic. Telemed J e-Health. https://doi.org/10.1089/tmj.2020.0219

7. Burki TK (2020) Cancer guidelines during the COVID-19 pandemic. Lancet Oncol 21:629-630

8. Sirintrapun SJ, Lopez AM (2018) Telemedicine in cancer care. Am Soc Clin Oncol Educ Book 38:540-545

9. ASCO (2020) ASCO Special report: guide to cancer care delivery during the COVID-19 pandemic

10. Chen CC, Yamada T, Smith J (2014) An evaluation of healthcare information on the Internet: the case of colorectal cancer prevention. Int J Environ Res Public Health 11:1058-1075 
11. Shim M, Kelly B, Hornik R (2006) Cancer information scanning and seeking behavior is associated with knowledge, lifestyle choices, and screening. J Health Commun 11(Suppl 1):157-172

12. Finney Rutten LJ, Agunwamba AA, Wilson P et al (2016) Cancerrelated information seeking among cancer survivors: trends over a decade (2003-2013). J Cancer Educ 31:348-357

13. Shea-Budgell MA, Kostaras X, Myhill KP, Hagen NA (2014) Information needs and sources of information for patients during cancer follow-up. Curr Oncol 21:165-173

14. Effenberger M, Kronbichler A, Shin JI, Mayer G, Tilg H, Perco P (2020) Association of the COVID-19 pandemic with Internet search volumes: a Google TrendsTM analysis. Int J Infect Dis 95:192-197

15. Eysenbach G (2011) Infodemiology and infoveillance: tracking online health information and cyberbehavior for public health. Am J Prev Med 40:S154-S158

16. Cook S, Conrad C, Fowlkes AL, Mohebbi MH (2011) Assessing Google flu trends performance in the United States during the 2009 influenza virus A (H1N1) pandemic. PLoS ONE 6:e23610

17. Kandula S, Shaman J (2019) Reappraising the utility of Google Flu Trends. PLoS Comput Biol 15:e1007258

18. Kumar N, Pandey A, Garg N, Sampene E, Lavie CJ (2018) Seasonal and geographic patterns in seeking cardiovascular health information: an analysis of the online search trends. Mayo Clin Proc 93:1185-1190

19. Mavragani A (2020) Infodemiology and infoveillance: scoping review. J Med Internet Res 22:e16206

20. Schootman M, Toor A, Cavazos-Rehg P et al (2015) The utility of Google Trends data to examine interest in cancer screening. BMJ Open 5:e006678

21. Phillips CA, Barz Leahy A, Li Y, Schapira MM, Bailey LC, Merchant RM (2018) Relationship between state-level Google online search volume and cancer incidence in the United States: retrospective study. J Med Internet Res 20:e6

22. Siegel RL, Miller KD, Jemal A (2020) Cancer statistics, 2020. Cancer J Clin 70:7-30

23. Google (2020) Google Trends Help. https://support.google.com/ trends/. accessed $08 \mathrm{Jul} 2020$.

24. Rosenkrantz AB, Prabhu V (2016) Public interest in imagingbased cancer screening examinations in the United States: analysis using a web-based search tool. AJR Am J Roentgenol 206:113-118

25. Islami F, Goding Sauer A, Miller KD et al (2018) Proportion and number of cancer cases and deaths attributable to potentially modifiable risk factors in the United States. CA Cancer J Clin $68: 31-54$
26. The LO (2020) Safeguarding cancer care in a post-COVID-19 world. Lancet Oncol 21:603

27. Giannakoulis VG, Papoutsi E, Siempos II (2020) Effect of cancer on clinical outcomes of patients with COVID-19: a meta-analysis of patient data. JCO Glob Oncol 6:799-808

28. Mehta V, Goel S, Kabarriti R et al (2020) Case fatality rate of cancer patients with COVID-19 in a New York hospital system. Cancer Discov 10:935-941

29. Williamson EJ, Walker AJ, Bhaskaran K et al (2020) Factors associated with COVID-19-related death using OpenSAFELY. Nature 584:430-436

30. Yu J, Ouyang W, Chua MLK, Xie C (2020) SARS-CoV-2 transmission in patients with cancer at a tertiary care hospital in Wuhan. China JAMA Oncol 6:1108-1110

31. Lewis MA (2020) Between scylla and charybdis - oncologic decision making in the time of Covid-19. N Engl J Med 382:2285-2287

32. DeRosier J (2020) Sharp declines in new cancer diagnoses observed during COVID-19 pandemic

33. Maringe C, Spicer J, Morris M et al (2020) The impact of the COVID-19 pandemic on cancer deaths due to delays in diagnosis in England, UK: a national, population-based, modelling study. Lancet Oncol 21:1023-1034

34. Sud A, Jones M, Broggio J et al (2020) Collateral damage: the impact on outcomes from cancer surgery of the COVID-19 pandemic. Ann Oncol 31:1065-1074

35. Ricciardiello L, Ferrari C, Cameletti M et al (2020) Impact of SARS-CoV-2 pandemic on colorectal cancer screening delay: effect on stage shift and increased mortality. Clin Gastroenterol Hepatol. https://doi.org/10.1016/j.cgh.2020.09.008

36. Calabrò L, Peters S, Soria JC et al (2020) Challenges in lung cancer therapy during the COVID-19 pandemic. Lancet Respir Med 8:542-544

37. Passaro A, Peters S, Mok TSK, Attili I, Mitsudomi T, de Marinis F (2020) Testing for COVID-19 in lung cancer patients. Ann Oncol 31:832-834

38. Paterson C, Bacon R, Dwyer R et al (2020) The role of telehealth during the COVID-19 pandemic across the interdisciplinary cancer team: implications for practice. Semin Oncol Nurs 36:151090

Publisher's Note Springer Nature remains neutral with regard to jurisdictional claims in published maps and institutional affiliations. 